\title{
A fast integrated mobility spectrometer for rapid measurement of sub-micrometer aerosol size distribution, Part I: Design and model evaluation
}

\author{
Jian Wang ${ }^{\mathrm{a}, *}$, Michael Pikridas ${ }^{\mathrm{a}, 1}$, Steven R. Spielman ${ }^{\mathrm{b}}$, Tamara Pinterich \\ a Environmental and Climate Sciences Department, Brookhaven National Laboratory, Upton, New York 11973, USA \\ b Aerosol Dynamics Inc., Berkeley, California 94710, USA
}

\section{A R T I C L E I N F O}

\section{Keywords:}

Fast Integrated Mobility Spectrometer

Aerosol size distribution

High time resolution

dynamic size range

Electrical mobility

Mobility resolution

\begin{abstract}
A B S T R A C T
A fast integrated mobility spectrometer (FIMS) was previously developed to characterize submicron aerosol size distributions at a frequency of $1 \mathrm{~Hz}$ and with high size resolution and counting statistics (Kulkarni \& Wang, 2006a, 2006b; Olfert, Kulkarni, \& Wang, 2008). However, the dynamic size range of the FIMS was limited to one decade in particle electrical mobility. It was proposed that the FIMS dynamic size range can be greatly increased by using a spatially varying electric field (Wang, 2009). This electric field creates regions with drastically different field strengths in the separator, such that particles of a wide diameter range can be simultaneously classified and subsequently measured. A FIMS incorporating this spatially varying electric field is developed. This paper describes the theoretical frame work and numerical simulations of the FIMS with extended dynamic size range, including the spatially varying electric field, particle trajectories, activation of separated particles in the condenser, and the transfer function, transmission efficiency, and mobility resolution. The influences of the particle Brownian motion on FIMS transfer function and mobility resolution are examined. The simulation results indicate that the FIMS incorporating the spatially varying electric field is capable of measuring aerosol size distribution from 8 to $600 \mathrm{~nm}$ with high time resolution. The experimental characterization of the FIMS is presented in an accompanying paper.
\end{abstract}

\section{Introduction}

Atmospheric aerosols play critical roles in air quality, public health, and visibility. In addition, they strongly influence climate by scattering solar radiation and by changing the reflectivity and lifetime of clouds. Real-time measurement of sub-micrometer aerosol size distribution is important in studies of aerosol properties and processes. Fast measurement is often necessary to capture transient aerosol dynamics, including those during nucleation and new particle formation processes. In addition, for measurements onboard mobile platforms such as research aircraft, high time resolution is often required to capture the spatial and temporal variations of atmospheric aerosols.

Existing techniques and instruments for measuring sub-micrometer aerosol size distributions are summarized in Kulkarni and Wang (2006a) and are briefly described here. Sub-micrometer aerosol size distributions are traditionally measured using Scanning Mobility Particle Sizers (SMPS) (Wang \& Flagan, 1990). There have been many recent advancements in SMPS, including increased

\footnotetext{
* Corresponding author.

E-mail address: jian@bnl.gov (J. Wang).

${ }^{1}$ Current affiliation: The Cyprus Institute, Environment Energy and Water Research Center, Nicosia, Cyprus.
} 
size resolution in nanometer size range (Chen, Pui, Hummes, Fissan, Quant \& Sem, 1998; Flagan, 2004; Jiang et al., 2011; Rosser \& de la Mora, 2005; Song \& Dhaniyala, 2007), improved time resolution (Han, Chen, Pui, \& Anderson, 2000; Wang, McNeill, Collins, \& Flagan, 2002), and portability (Barmpounis, Maisser, Schmidt-Ott, \& Biskos, 2016; Flagan, 2004; Steer, Gorbunov, Muir, Ghimire, \& Rowles, 2014). Because SMPS measures one particle size at a time, the classifying voltage needs to be scanned over a wide range in order to cover the entire aerosol size distribution. This voltage scan typically takes a minimum of $60 \mathrm{~s}$ and is too slow for many applications (i.e., measurements onboard research aircraft). Electrical mobility-based instruments also include Electrical Aerosol Spectrometer (EAS, Tammet, Mirme, \& Tamm, 2002), Engine Exhaust Particle Sizer (EEPS, Johnson, Caldow, Pocher, Mirme, \& Kittelson, 2004; Wang, Storey, Domingo, Huff, Thomas \& West, 2006), and Differential Mobility Spectrometer (DMS, Biskos, Reavell, \& Collings, 2005), which measure particles of different mobilities simultaneously using a series of integrated electrometers. These instruments are capable of rapid measurement of aerosol size distribution, but their applications have largely been limited to aerosols with very high number concentrations (e.g., engine exhausts) due to the low sensitivity of electrometers. Besides low sensitivity, the size resolutions of EAS, EEPS, and DMS are considerably lower than that of SMPS. Recent developments in electrical mobility based instruments also include an Aspirating Drift Tube Ion Mobility Spectrometer capable of measuring particle size distribution from 2 to $\sim 12 \mathrm{~nm}$ within several seconds (Oberreit, McMurry, \& Hogan, 2014).

A Fast Integrated Mobility Spectrometer (FIMS) was developed previously for rapid measurements of submicron aerosol size distribution under typical ambient concentrations (Kulkarni \& Wang, 2006a, 2006b). Inside the FIMS, charged particles of different electrical mobilities are first separated by an electric field into different flow streamlines. The spatially separated particles are subsequently grown into super-micrometer droplets inside a condenser. The grown droplets are illuminated by a sheet of laser light and imaged by a CCD camera. In addition to the number of particles, the images also provide particle position and therefore the distance travelled by particle in the direction of the electric field, which directly relates to the particle mobility. By counting particles of different electrical mobilities simultaneously, the FIMS measures aerosol size distribution within $1 \mathrm{~s}$, about two orders of magnitude faster than traditional SMPS. Because individual particles are detected optically, the FIMS also provides measurements with significantly higher signal to noise ratio compared to electrometer based systems. The performance of the FIMS was characterized, and a data inversion technique was developed to derive size distribution from FIMS data (Kulkarni \& Wang, 2006b; Olfert, Kulkarni \& Wang, 2008; Olfert \& Wang, 2009). While capable of rapid measurement, the FIMS presented by Kulkarni and Wang (2006a, 2006b) has a narrow dynamic size range limited to a factor of 3 to 5 in particle diameter (i.e., a factor of 10 in electrical mobility). As a result, measurements of sub-micrometer size distribution (e.g., 15 to $1000 \mathrm{~nm}$ ) require three FIMS units operated in parallel with different separating voltages to cover the entire size range.

It was proposed that the dynamic size range of the FIMS can be increased without loss in size and time resolutions by using a spatially varying electric field in the separator (Wang, 2009). This electric field creates regions with drastically different field strengths in the separator, such that particles of a wide diameter range can be simultaneously classified and subsequently measured. This is equivalent to combining into a single unit several original FIMS, operated with different separating voltages. A FIMS incorporating this spatially varying electric field is developed. Here we describe the theoretical framework and numerical simulations of the FIMS characteristics, including the spatially varying electric field, particle trajectories, and activation of particles inside the condenser. The dynamic size range, transmission efficiency, and transfer function of the FIMS are derived from the simulations and discussed. The influences of the particle Brownian motion on transfer function and mobility resolution are examined. The experimental characterization of the FIMS is reported in an accompanying paper.

\section{Instrument Design}

The principle of the FIMS operating with a spatially varying electric field is essentially the same as that of the original version described in Kulkarni and Wang (2006a), and is briefly described below. The FIMS has three major sections - (i) separator, (ii) condenser, and (iii) detector, where are arranged sequentially to form a continuous rectangular channel. A schematic of the FIMS is present in Fig. 1 of Wang (2009). Particle-free sheath flow saturated with n-heptanol enters the separator from a top entrance. Charged particles are carried into the separator by a smaller aerosol flow through a narrow tangential slit. Inside the separator, the charged particles are separated by the electric field into mobility-dependent trajectories. The spatially separated particles subsequently enter the condenser. As no electric field is applied in the condenser, particle positions in the channel cross section (i.e., $x$ and $y$ coordinates) essentially remain unchanged downstream of the separator. The separated particles grow into supermicrometer droplets in a supersaturated environment inside the condenser. At the exit of the condenser, the grown droplets are illuminated by a laser sheet and imaged by a high-speed CCD camera. The images provide the position of individual particles, which directly relates to the particle mobility. As a result, particles of different mobilities/sizes can be measured simultaneously. The original FIMS reported in Kulkarni and Wang (2006a and 2006b) employs a uniform electric field, and its dynamic size range is limited to a factor of 10 in electrical mobility in order to maintain a good size resolution (i.e., mobility resolution no less than 5).

The development of the FIMS with improved dynamic size range involves two major modifications to the original FIMS. First, the original HV electrode, a solid aluminum plate, is replaced. Wang (2009) proposed an electrode where voltage applied varies exponentially as a function of $y$ position (Note the coordinate system depicted in Fig. 1 of Wang (2009) is used in this study). However, such an electrode is very difficult to construct, if possible at all. Instead, a thick printed circuit board (PCB) with 61 straight copper traces running in the direction of the flow (z-direction) is used as the electrode. The diagram of this new electrode in the $x-y$ plane (i.e., cross section of the separator channel) is shown in Fig. 1 . The minimum voltage $\left(V_{1}\right)$ of $20 \mathrm{~V}$ and maximum voltage $\left(V_{2}\right)$ of $8000 \mathrm{~V}$ are applied to the first and last traces, which have a width of $3 \mathrm{~cm}$ and $2 \mathrm{~cm}$, respectively. Fifty-nine thin traces of $0.15 \mathrm{~mm}$ in width are evenly spaced over a distance of $6 \mathrm{~cm}$ in the center (i.e., $-3 \mathrm{~cm} \leq y \leq 3 \mathrm{~cm}$ ). Individual voltages ranging from 20 to 


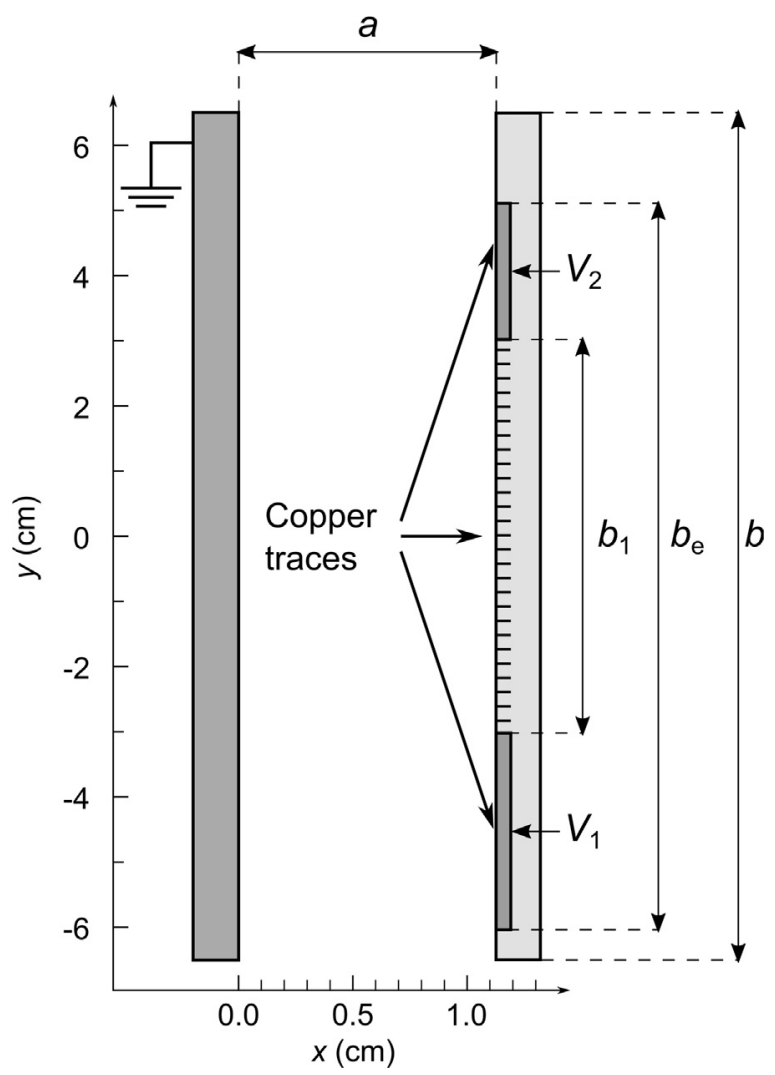

Fig. 1. Schematic of the electrodes used to create the spatially varying electric field in the FIMS separator.

$8000 \mathrm{~V}$ are generated by a network of resistor dividers, and applied to these 59 thin traces. The voltage variation as a function of $y$ position is shown in Fig. 2. The applied voltage increases approximately exponentially with the $y$ position of thin traces from 20 to $1800 \mathrm{~V}$, then increases linearly with $y$ from 1800 to $8000 \mathrm{~V}$. This is to reduce the voltage differences between adjacent traces and to avoid arcing in the high voltage region. Only particles detected within the viewing window (i.e., $0.2 a \leq x \leq 0.8 a,-3.5 \mathrm{~cm} \leq y \leq$ $3.5 \mathrm{~cm}$, where $a=1.12 \mathrm{~cm}$, and represents the gap between ground and HV electrodes) at the center of the channel cross section are used for measurements, in order to avoid the edge effects of the electric and flow fields, and to maintain good mobility resolution (Kulkarni \& Wang, 2006a, 2006b). Note the voltage is negative to separate positively charged particles, and the voltages presented here are absolute values. The second major change is the increase of the length of the electrode $\left(l_{s}\right)$, therefore the separator. This is to

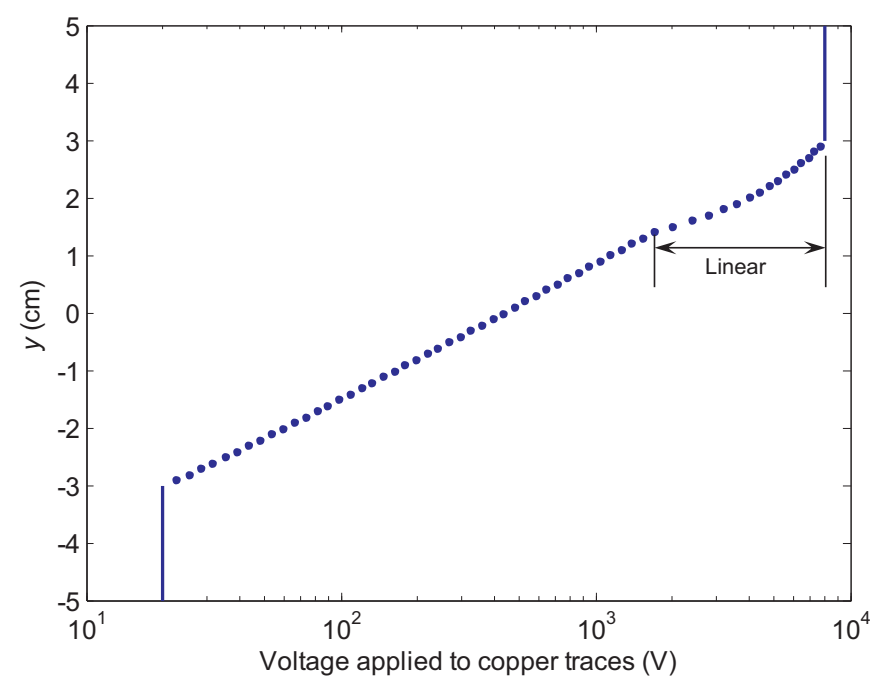

Fig. 2. Variation of the voltage applied to copper traces as a function of trace $y$ position. 
Table 1

Physical dimensions and parameters of the modified FIMS employing a spatially varying electric field.

\begin{tabular}{lll}
\hline Description & Symbol & Values \\
\hline Distance between separator electrodes & $a$ & $1.12 \mathrm{~cm}$ \\
Width of separator & $b$ & $12.70 \mathrm{~cm}$ \\
Length of the separating region & $l_{s}$ & $17.43 \mathrm{~cm}$ \\
Minimum Voltage & $V_{1}$ & $20 \mathrm{~V}$ \\
Maximum Voltage & $V_{2}$ & $8000 \mathrm{~V}$ \\
Width of the HV electrode (Fig. 1) & $b_{e}$ & $11.0 \mathrm{~cm}$ \\
Width of the area with 59 thin traces (Fig. 1) & $b_{1}$ & $6.0 \mathrm{~cm}$ \\
Sheath flow rate & $Q_{s h}$ & $13 \mathrm{~L} \mathrm{~min}-1$ \\
Aerosol flow rate & $Q_{a}$ & $0.26 \mathrm{~L} \mathrm{~min}-1$ \\
Viewing window $x$-coordinate range & & $0.2 a$ to $0.8 a$ \\
Viewing window $y$-coordinate range & & -3.5 to $3.5 \mathrm{~cm}$ \\
\hline
\end{tabular}

increase the upper limit of particle size that could be measured by the FIMS. Key physical dimensions and the operating parameters of the modified FIMS are given in Table 1.

\section{Simulation of the characteristics of FIMS with enhanced dynamic size range}

\subsection{Spatially varying electric Field}

The characteristics of the FIMS are examined using simulated particle trajectories inside the instrument. The simulation of the particle trajectories requires the knowledge of the electric field inside the separator. Unlike the idealized cases described in Wang (2009), the electric field created by the new electrodes shown in Fig. 1 cannot be derived analytically, and was simulated using COMSOL Multiphysics ${ }^{\mathrm{TM}}$. As the length of the electrodes $\left(l_{s}\right)$ is much greater than the gap between the two opposing electrodes $(a)$, the electric field was assumed as constant along the $z$ direction (direction of the flow). The simulated 2-D electric field was then
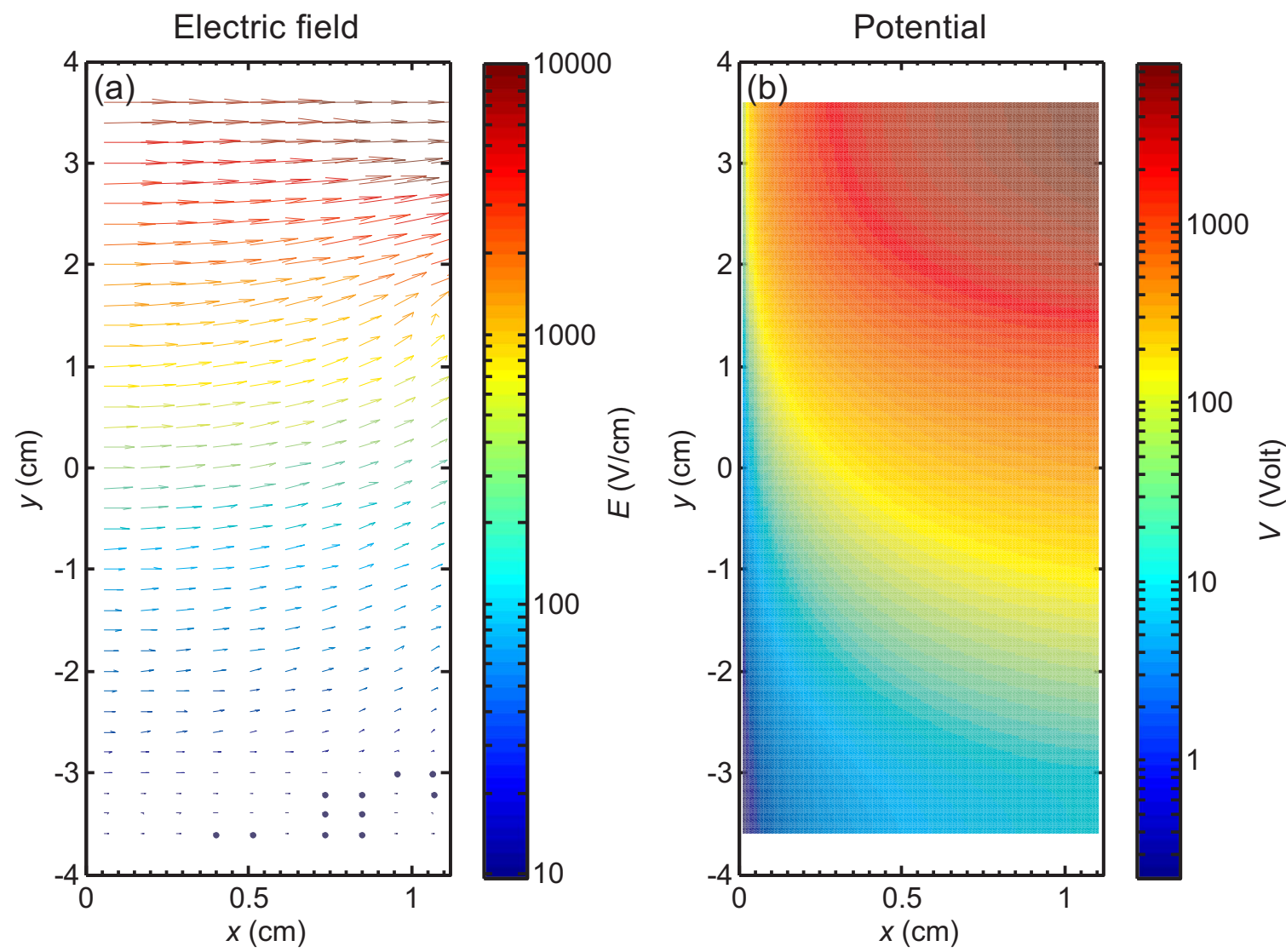

Fig. 3. Simulated 2-D (a) electric field and (b) potential shown in $x$-y plane. Both the length and the color of the field vector in plot (a) scale with the logarithm of the electric field strength. The ground and HV electrodes are at $x=0$ and $1.12 \mathrm{~cm}$, respectively. 
imported into MATLAB ${ }^{\mathrm{TM}}$ and interpolated to provide values at any given point in $x-y$ space. Figs. 3a and b show the simulated 2-D electric field and potential, respectively. The strength of the electric field varies by a factor of $\sim 500$ (Fig. 3a). The electric field in the regions of $y<-3.0 \mathrm{~cm}$ and $y>3.0 \mathrm{~cm}$ is largely uniform and has no appreciable $y$-component, due to constant voltages applied to the both ends of the HV electrode. In contrast, the electric field in the center of the separator cross section has a large $y$-component due to the strong spatial variation in applied voltage. These features are consistent with those of idealized electric field presented in Wang (2009).

\subsection{Simulation of particle trajectories inside FIMS}

The particle trajectories inside the FIMS were simulated using the simulated $2 \mathrm{D}$ electric field described above and assuming a well-developed 1-D flow field in the $z$ direction:

$$
u_{z}=\frac{6 Q_{t}}{a^{3} b} x(a-x)
$$

where $Q_{t}$ is effective total volumetric flow rate for the 1-D flow, and is given by:

$$
Q_{t}=c\left(Q_{s h}+Q_{a}\right)
$$

The factor $c$ is included because the edge effects at the side walls of the separator (i.e., $y= \pm 0.5 b$ ) are neglected in the 1-D flow field. As the actual flow velocity near the side walls is lower, the flow velocity in the center region, where particles are detected and counted (i.e., $-3.5 \mathrm{~cm}<y<3.5 \mathrm{~cm}$ ), is higher than that assuming no edge effects. The adjustment factor $c$ depends on the dimension of the separator cross section, and has a value of 1.056 for this study (Olfert et al., 2008). The motion of particles inside the FIMS was simulated using the Langevin equation (Ermak, 1975),

$$
\mathbf{r}(t+\Delta t)=\mathbf{r}(t)+\left[u_{z} \mathbf{k}+Z_{p}\left(D_{p}, \varphi, T, P\right)\left(E_{x}(\mathbf{r}) \mathbf{i}+E_{y}(\mathbf{r}) \mathbf{j}\right)\right] \Delta t+\delta \mathbf{r}_{G}
$$

where $\mathbf{r}(t)$ and $\mathbf{r}(t+\Delta t)$ represent the particle's position at time $t$ and $t+\Delta t$, respectively. $E_{x}$ and $E_{y}$ are the $x$ and $y$ components, respectively, of the simulated 2-D electric field described above. $Z_{p}$ is the electrical mobility:

$$
Z_{p}=\frac{q C_{c}}{3 \pi \mu D_{p}}
$$

where $q$ is the electric charge on the particle, $C_{c}$ is the Cunningham slip correction factor, $\mu$ is the viscosity of the suspending medium (i.e., air), and $D_{p}$ is the particle diameter. In this study, the particle mobility is calculated at a temperature of $298.15 \mathrm{~K}$ and a pressure of $1013.25 \mathrm{hPa}$. The term $\delta \mathbf{r}_{\mathrm{G}}$ in Eq. (3) corresponds to the particle Brownian displacement, and was simulated individually for each
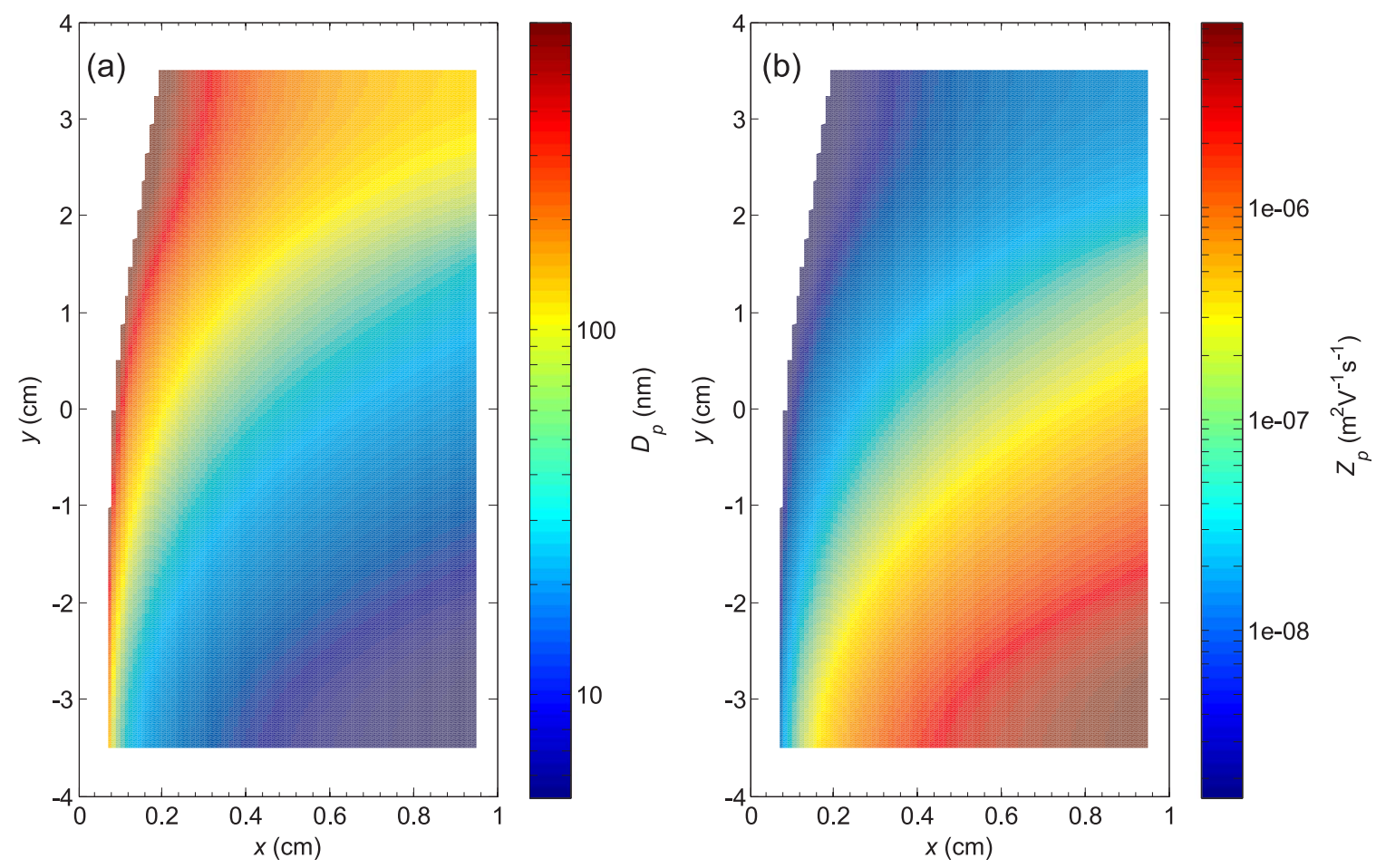

Fig. 4. The $x-y$ positions at the separator exit for non-diffusing particles introduced along the central aerosol inlet flow streamline, colored by (a) particle diameter and (b) electrical mobility. 
particle by employing a Gaussian-shaped random number generator of zero mean and standard deviation equal to $2 D \Delta$ t. $D$ is the particle diffusion coefficient, and is related to particle mobility by $D=Z_{p} k_{B} T / q$, where $k_{B}$ is the Boltzmann constant, and $T$ is the temperature of the suspending medium.

\subsection{Instrument response mobility of FIMS}

As the FIMS measures a particle's mobility from its detected position (i.e., $x$ and $y$ coordinates) at the exit of condenser, an instrument response mobility is defined following the same approach as in Kulkarni and Wang (2006a), Wang (2009) to facilitate the analysis of FIMS characteristics. Let $\Psi_{1, \text { in }}$ and $\Psi_{2, \text { in }}$ represent the two streamlines that bound the aerosol flow at the inlet (Kulkarni \& Wang, 2006a), for a non-diffusing particle enters the separator along the central inlet flow streamline, $\Psi_{c, \text { in }}=\frac{\Psi_{1, \text { in }}+\Psi_{2, \text { in }}}{2}$, the instrument response mobility, $Z_{p}^{*}$, at the $x-y$ position where the particle is detected is the same as the particle mobility $Z_{p}$.

Instrument response mobility $\left(Z_{p}{ }^{*}\right)$ was derived by simulating the trajectories of singly charged, non-diffusion particles introduced at 1000 initial $y$ positions $\left(y_{i n}\right)$ evenly spaced from -4.0 and $4.0 \mathrm{~cm}$ along the central aerosol flow stream line. Particles introduced outside this $y$ range exit the condenser outside the viewing window (Table 1 ). The initial $x$ position corresponding to the central inlet flow streamline, $x_{i n}$ was derived from the following equation:

$$
b \cdot \int_{0}^{x_{i n}} u_{z}(x) d x=\int_{0}^{x_{i n}} \frac{6 Q_{t}}{a^{3}} x(a-x) d x=\frac{1}{2} Q_{a} .
$$

At each $y_{\text {in }}$ position, particles of 2500 different diameters logarithmically distributed from 4 to 900 nm were introduced, and their positions at the exit of the separator were determined by simulating particle trajectories assuming no Brownian diffusion. For nondiffusing particles, their $x-y$ positions at the separator exit are the same as those detected following condensational growth inside the condenser, where there is no electric field.

Fig. 4 shows the $x-y$ positions of these particles at the separator exit, colored by particle diameter (Fig. 4a) and electrical mobility (Fig. 4b). By definition, Fig. 4b shows the map of instrument response mobility in the FIMS channel cross section. As expected, particles of low electrical mobility (large particles) are classified in the upper region of the cross section where the electric field is stronger, while in the lower region they remain close to the ground electrode, where they were initially introduced. Particles of high electrical mobility (small particles) in the upper region travel too fast and, they either are lost on the HV electrode or exit the separator outside the viewing window. Small particles are instead classified in the lower region where the field is weaker (Fig. 4).

\subsection{FIMS transfer function}

The FIMS transfer function $P\left(Z_{p}, Z_{p}^{*}\right)$ follows the same definition as in Kulkarni and Wang (2006a), Wang (2009), and is described as follows. The probability of a particle with mobility $Z_{p}$ introduced into the separator and being measured by the FIMS with instrument response mobility from $Z_{p}^{*}$ to $Z_{p}^{*}+d Z_{p}^{*}$ is $P\left(Z_{p}, Z_{p}^{*}\right) d Z_{p}^{*}$. As shown later, $P\left(Z_{p}, Z_{p}^{*}\right)$ often peaks at $Z_{p}{ }^{*}=Z_{p}$. To facilitate the comparison of transfer functions for different $Z_{p}$, we adopt a slightly different form of transfer function, $P\left(Z_{p}, Z_{p}^{*} / Z_{p}\right)$. The two forms of the transfer function are related to each other by $P\left(Z_{p}, Z_{p}^{*} / Z_{p}\right)=Z_{p} \cdot P\left(Z_{p}, Z_{p}^{*}\right)$. When particle Brownian diffusion is taken into consideration, the transfer function also depends on the number of electric charges carried by particles. For example, a doubly charged particle with the same electrical mobility is larger therefore has a lower diffusivity than that of a singly charged particle. Here the transfer function is derived based on singly charged particles. The derivation of the transfer function for multiple-charged particles is very similar.

For the derivation of the FIMS transfer function, particle trajectories were simulated for particles introduced at 1000 initial $y$ positions evenly spaced from -4.0 to $4.0 \mathrm{~cm}$, and at 100 initial $x$ positions evenly distributed in the inlet flow streamline space between $\Psi_{1, i n}$ and $\Psi_{2, i n}$ that bound the aerosol flow. The initial $x$ positions $\left(x_{i n, j}, 0 \leq j \leq 99\right)$ were derived using the following equation (Wang, 2009):

$$
b \cdot \int_{0}^{x_{i n, j}} u_{z}(x) d x=\int_{0}^{x_{i n, j}} \frac{6 Q_{t}}{a^{3}} x(a-x) d x=\frac{(j+0.5)}{100} Q_{a}, 0 \leq j \leq 99 .
$$

Altogether, particles were introduced at a total of 100,000 initial positions. At each position, the simulation of particle trajectories was repeated 10 times. Therefore a total of 1,000,000 particle trajectories were simulated for each particle diameter. While no electric field is applied outside the separator, particle position in the $x-y$ plane may continue to vary due to Brownian motion, especially for small particles. Therefore, the simulation of particle trajectory continued downstream of the end of the electrode, to account for particle Brownian motion before activation inside the condenser. Once activated, particles quickly grown to a sufficiently large size (e.g., a few hundred nanometers) such that particle Brownian motion becomes negligible, and the particle $x$-y position essentially remains unchanged afterwards. The distance traveled by a particle between the end of electrode and the point of its activation $\left(L_{a}\right)$ depends on particle size and the supersaturation profile along the particle trajectory, therefore the particle position within the channel cross section (i.e., $x$ - $y$ plane). The detected particle position was simulated using fixed $L_{a}$ of 5.9 and $13.5 \mathrm{~cm}$, representing the minimum and maximum values, respectively. The value of $5.9 \mathrm{~cm}$ includes a total length of $4.6 \mathrm{~cm}$ from end of the electrode to the end of the separator and a $1.3 \mathrm{~cm}$ thick thermal insulation flange between the separator and condenser, and represents the distance between the end of the HV electrode and the beginning of the condenser. The value of $13.5 \mathrm{~cm}$ includes an additional distance of $7.6 \mathrm{~cm}$ into the condenser, which presents the longest distance that a particle travels in the condenser before activation ( $8 \mathrm{~nm}$ particles with trajectories near $x=0.5 a$, Fig. $8 \mathrm{~b}$ ). The simulations using the two $L_{a}$ values provide the lower and 

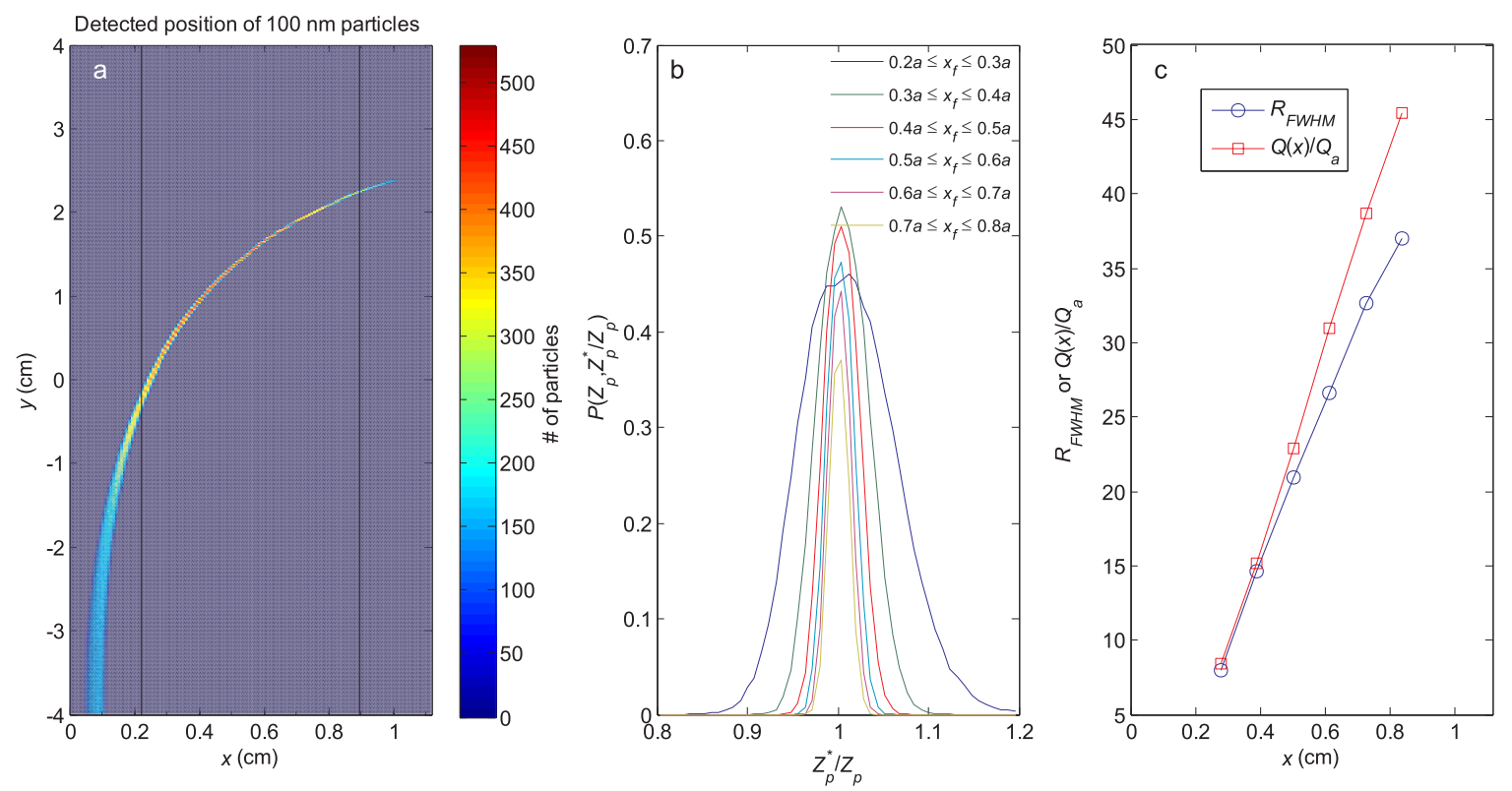

Fig. 5. (a) Detected position of particles with diameter of $100 \mathrm{~nm}$ simulated with $L_{a}=5.9 \mathrm{~cm}$. The color represents the number of particles within a 0.08 mm $\times 0.08$ mm pixel. (b) Transfer function of $100 \mathrm{~nm}$ particles detected within subareas of the viewing window. (c) Resolution $R_{F W H M}$ and the ratio of flow between the ground electrode and particle detection position (i.e., $Q(x)$ ) to aerosol flow rate.

upper limits of the impact of particle Brownian diffusion on the FIMS transfer function. After the detected particle $x$ - $y$ positions were simulated, they were converted to instrument response mobility $Z_{p}^{*}$ using the map shown in Fig. 4b through interpolation. The transfer function $P\left(Z_{p}, Z_{p}^{*} / Z_{p}\right)$ is simply the histogram of $Z_{p}{ }^{*} / Z_{p}$ for particles detected within the viewing window, normalized by the number of the simulated particle trajectories and the width of $Z_{p}{ }^{*} / Z_{p}$ bin, and scaled by a factor of $8 / 12.7$. This factor is necessary because aerosol flow is introduced into the separator over a length of $12.7 \mathrm{~cm}$ (in $y$ direction) while in our simulations, particles are introduced only within $8 \mathrm{~cm}$ width at the center of the channel.

The detected positions of particles with diameter of $100 \mathrm{~nm}$ simulated using $L_{a}$ of $5.9 \mathrm{~cm}$ are present in Fig. 5. Depending on its initial $y$ position introduced at the aerosol inlet, particles of same mobility experience electric forces of different strengths, leading to a range of final $x$ (and $y$ ) positions detected. Here we use $x_{f}$ and $y_{f}$ to denote the final detected particle $x$ and $y$ positions, respectively. Using the same approach described above, the transfer functions for particles detected within subareas of the viewing window were derived to gain insights into the mobility resolution of the FIMS. Fig. $5 \mathrm{~b}$ and c shows the transfer function and corresponding mobility resolution $R_{F W H M}$, defined as the ratio of the particle mobility $\left(Z_{p}\right)$ to the full width at half maximum of the transfer function (Eq. 28 of Kulkarni \& Wang, 2006a), for $100 \mathrm{~nm}$ particles detected in different subareas. The transfer function is narrower and the resolution is higher for particles that migrate further in the $x$ direction under a stronger electrical field. The value of $R_{F W H M}$ is consistent with the ratio of the rate of flow between the ground electrode (i.e., $x=0$ ) and particle $x$ position to the aerosol flow rate, except in area

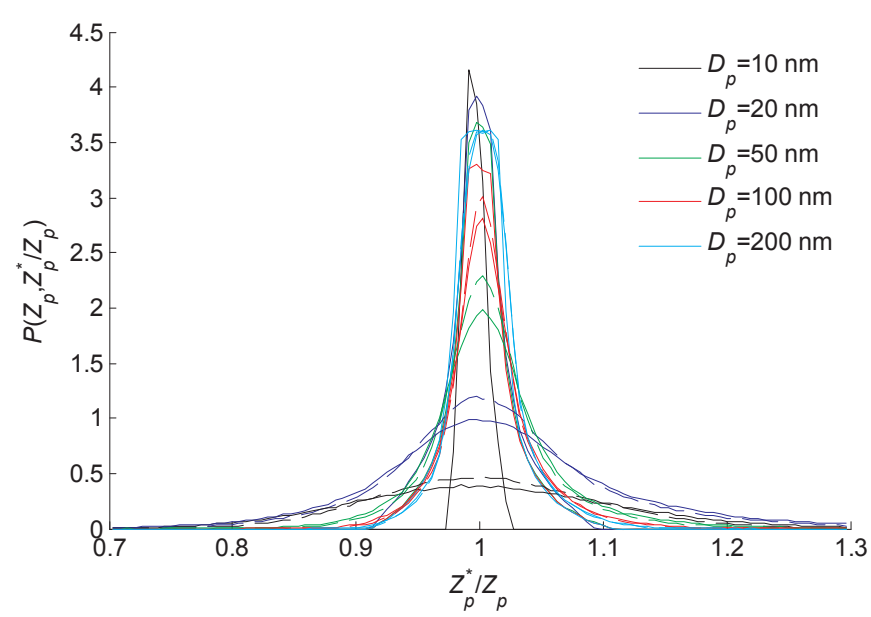

Fig. 6. Simulated FIMS transfer functions for particles with diameters of 10, 20, 50, 100, and 200 nm. The line colors correspond to different particle diameter. The solid, dashed, and dot lines represent simulations assuming no particle diffusion, and particle diffusion with $L_{a}$ of 5.9 and $13.5 \mathrm{~cm}$, respectively. 

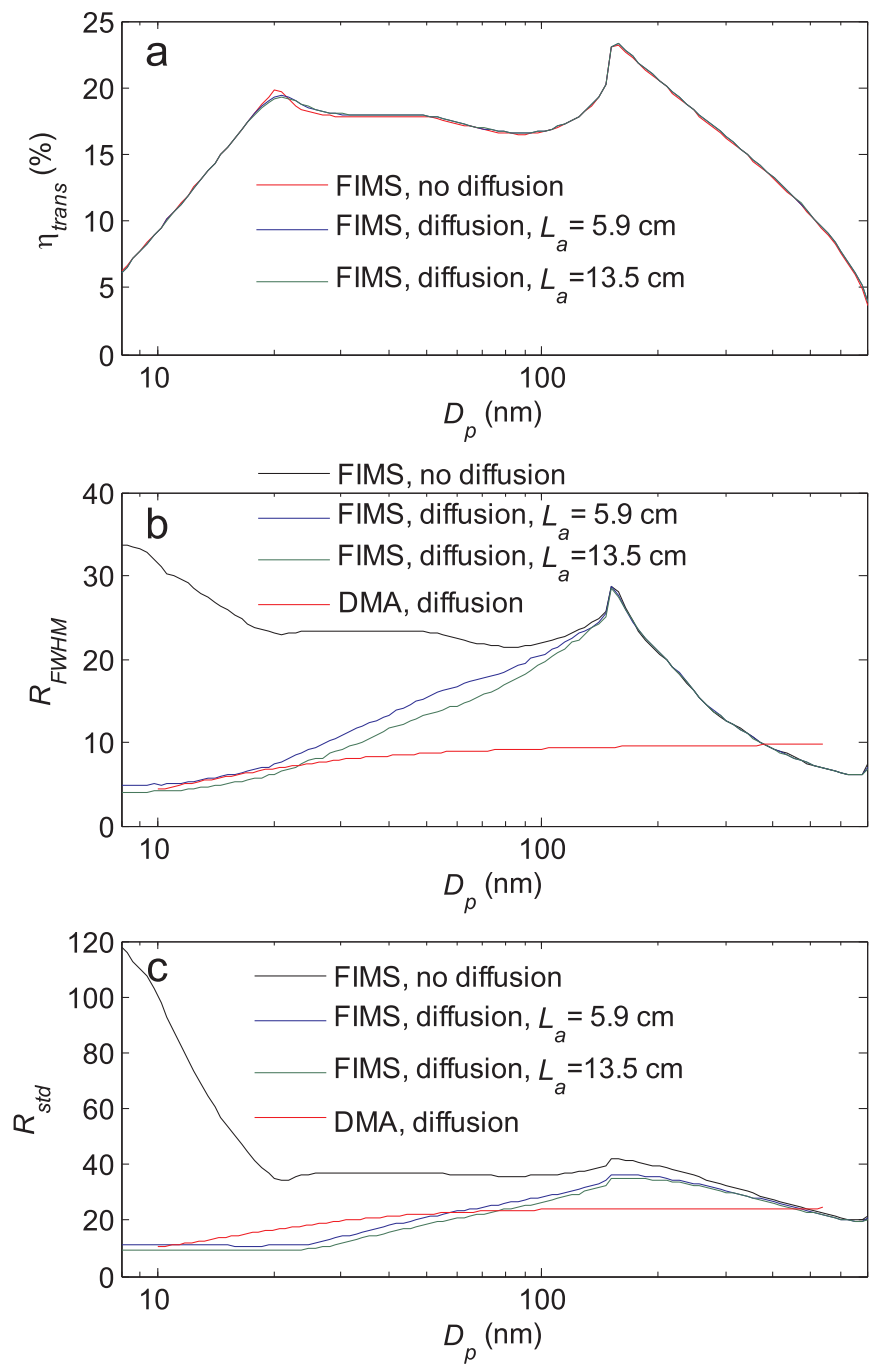

Fig. 7. Simulated FIMS transmission efficiency ( $\eta_{\text {trans }}$, panel a), resolution based on full width at half maximum ( $R_{F W H M}$, panel b), and resolution based on the standard deviation ( $R_{\text {std }}$, panel c) assuming no particle diffusion, and particle diffusion with $L_{a}$ of 5.9 and $13.5 \mathrm{~cm}$, respectively. Also shown in panel b and c are corresponding resolutions of a DMA (Model 3081, TSI Inc.) operated with $Q_{a}$ and $Q_{s h}$ of 0.5 and $5 \mathrm{~L} \mathrm{~min}^{-1}$, respectively.

with large $x$ values where resolution becomes limited by particle Brownian diffusion. This is consistent with $R_{F W H M}$ of FIMS employing an uniform electric field (Kulkarni \& Wang, 2006a) and the finding of Wang (2009), and suggests that measurements of high resolution may be achieved by using only particles detected in subareas with high $x$ values. The transfer function corresponding to the entire viewing window is simply the sum of the transfer functions for particles detected in the 6 subareas shown in Fig. $5 \mathrm{~b}$, and has an overall $R_{F W H M}$ value of 22.3 .

Fig. 6 shows the transfer function for particles with 5 diameters ranging from 10 to $200 \mathrm{~nm}$. Transfer functions were simulated assuming no particle diffusion, and particle diffusion with $L_{a}$ of 5.9 and $13.5 \mathrm{~cm}$, respectively. For non-diffusing particles, the transfer functions for 20,50,100, $200 \mathrm{~nm}$ particles are essentially the same, as the resolution for non-diffusing particles are largely determined by particle final $x$ position as discussed above (Kulkarni $\&$ Wang, 2006a; Wang, 2009). For these four sizes, particle final $x$ positions range from $0.2 a$ and $0.8 a$ within the viewing window. In comparison, the final $x$ position of particles with a diameter of $10 \mathrm{~nm}$ is greater than $0.35 a$ even in the region with the weakest electric field, leading to an overall narrower transfer function and a higher resolution. Particle Brownian diffusion has minor impact on the transfer function for particles with diameters of $100 \mathrm{~nm}$ or larger, but leads to substantial broadening of transfer function and therefore reduction in resolution when particle diameter is less than $50 \mathrm{~nm}$. It is worth noting that the difference in transfer functions simulated with the two different $L_{a}$ values is quite minor.

Fig. 7 shows the transmission efficiency $\left(\eta_{\text {trans }}\right)$, and resolutions based on full width at half maximum $\left(R_{F W H M}\right)$ and standard deviation $\left(R_{s t d}\right)$ as functions of particle diameter. The resolution $R_{s t d}$ is defined as the ratio of the particle mobility to the standard deviation of $Z_{p}^{*}$ calculated from the transfer function (i.e., Eq. 20 in Wang, 2009). The transmission efficiency represents the fraction of particles introduced into FIMS that have the final $x-y$ position within the viewing window, and is the integration of the transfer function over instrument response mobility: 


$$
\eta_{\text {trans }}=\int P\left(Z_{p}, Z_{p}^{*} / Z_{p}\right) d\left(Z_{p}^{*} / Z_{p}\right)
$$

The results are shown for simulations assuming no particle diffusion, and particle diffusion with $L_{\boldsymbol{a}}$ of 5.9 and $13.5 \mathrm{~cm}$, respectively. As expected, particle Brownian diffusion has negligible impact on $\eta_{\text {trans }}$. The transmission efficiency peaks at $\sim 20$ and $\sim 150 \mathrm{~nm}$, and shows a relatively constant value of $\sim 17 \%$ between. It decreases gradually below $20 \mathrm{~nm}$ and above $150 \mathrm{~nm}$, and reached $\sim 7 \%$ at 8 and $600 \mathrm{~nm}$. For non-diffusing particles, both $R_{F W H M}$ and $R_{s t d}$ decrease as particle diameter $D_{p}$ increases from 8 to $20 \mathrm{~nm}$, remain relatively constant before reaching a local maxima at $\sim 150 \mathrm{~nm}$, then decrease again from $\sim 150$ to $700 \mathrm{~nm}$. These variations of $\eta_{\text {trans, }}, R_{F W H M}$, and $R_{\text {std }}$ with $D_{p}$ are essentially the same as those reported in Wang (2009). The explanations for these variations are also detailed in Wang (2009). For example, the peak of $\eta_{\text {trans }}$ at $\sim 150 \mathrm{~nm}$ is due to the following reasons. At a given $D_{p}$, whether a particle can be detected within the viewing window or not largely depends on the strength of the electric field experienced by the particle in the separator. Because the strength of the electric field varies strongly with respect to $y$ coordinate, at a given $D_{p}$, only particles introduced within a certain range of initial $y$ position $\left(y_{i n}\right)$ are detected within the viewing window and contribute to $\eta_{\text {trans }}$. To a first order, $\eta_{\text {trans }}$ is proportional to this $y_{i n}$ range, which is referred to as the "effective" $y_{i n}$ range for the given $D_{p}$. At $D_{p}$ of $150 \mathrm{~nm}$, the effective $y_{i n}$ range is $0.53 \leq y_{i n} \leq 3.50 \mathrm{~cm}\left(\Delta y_{i n}=2.97 \mathrm{~cm}\right)$, which includes the entire region of the uniform strong electric field within the viewing window $\left(3.00 \leq y_{i n} \leq 3.50 \mathrm{~cm}\right.$, Fig. 2) as well as a region with spatially varying electric field $\left(0.53 \leq y_{\text {in }} \leq 3.00 \mathrm{~cm}\right)$. We note that $150 \mathrm{~nm}$ represents the lower diameter limit for particles that could be detected within the viewing window in the region of the uniform strong electric field. As $D_{p}$ decreases from $150 \mathrm{~nm}$, an increasing fraction of particles introduced in the region of $3.00 \leq y_{\text {in }} \leq 3.50 \mathrm{~cm}$ is detected outside of the viewing window or collected by the HV electrode, leading to reduced $\eta_{\text {trans }}$ (Wang, 2009). As $D_{p}$ increases above $150 \mathrm{~nm}$ and approaches the upper limit of the size measurement range, the effective $y_{i n}$ range and $\eta_{\text {trans }}$ also decrease because for particles near the upper limit of measurement range, only those introduced in the region with the strongest electric field are detected within the viewing window. For example, at a diameter of $400 \mathrm{~nm}$, the effective $y_{i n}$ range is reduced to $1.83 \leq y_{i n} \leq 3.50 \mathrm{~cm}$. The above variations in the effective $y_{i n}$ range lead to a peak in $\eta_{\text {trans }}$ at $\sim 150 \mathrm{~nm}$. Another contributing factor is that the electrode voltage varies linearly with $y$ in the region of $1.40 \leq y_{\text {in }}$ $\leq 3.00 \mathrm{~cm}$ (Fig. 2) instead of exponentially as in the region of $-3.00 \leq y_{i n} \leq 1.40 \mathrm{~cm}$. The relatively slow variation of voltage and electric field strength with $y$ also increases the effective $y_{\text {in }}$ range and therefore $\eta_{\text {trans }}$ near $150 \mathrm{~nm}$.

Particles with diameter of $150 \mathrm{~nm}$ that are introduced in the region of uniform strong electric field have high $x_{f}$ values (i.e., detected near the upper $x$ limit of the viewing window, $0.8 a$ ), corresponding to high mobility resolutions (Fig. 5). Due to the contribution of these particles with high $x_{f}$, the overall $R_{F W H M}$ and $R_{s t d}$ at $150 \mathrm{~nm}$ are higher than those of smaller particles with effective $y_{i n}$ range entirely within the center region where the electric field varies spatially. As $D_{p}$ further increases from $150 \mathrm{~nm}$, the $x_{f}$ for particles introduced within the uniform strong electric field region decreases, resulting in decreases of the overall $R_{F W H M}$ and $R_{\text {std }}$ (Wang, 2009).

The impact of particle Brownian diffusion on mobility resolutions is quite minor for particles with diameters greater than $\sim$ $100 \mathrm{~nm}$, but becomes increasingly significant as particle diameter decreases. For particles smaller than $20 \mathrm{~nm}$ in diameter, particle Brownian diffusion reduces $R_{F W H M}$ from above 28 to below 7 . There are minor differences between the resolutions based on simulations using the two different $L_{a}$ values, indicating the uncertainty in $L_{a}$ has a minor impact on simulated FIMS transfer function. The simulated FIMS resolutions are compared with those of a SMPS operated with a diameter range of 10-540 nm (i.e., a TSI model 3081 DMA operated with a sheath and aerosol flow rates of 5 and $0.5 \mathrm{~L} \mathrm{~min}^{-1}$, respectively, and classifying voltage scanned from 10 to $10,000 \mathrm{~V}$ ). Overall, the FIMS resolutions are generally comparable to those of the SMPS. The FIMS $R_{F W H M}$ is about the same as or better than that of the SMSP for particles with diameter less than $400 \mathrm{~nm}$. The FIMS $R_{\text {std }}$ is higher/lower than that of the SMPS for particles with diameters greater/less than $70 \mathrm{~nm}$. The generally higher resolutions of the FIMS, especially at large particle diameters, are mainly due to the higher $Q_{s h} / Q_{a}$ ratio of 50, compared to the ratio of 10 for typical SMPS.

\subsection{Activation of the particles inside the condenser}

The growth of the spatially separated particles in the condenser was simulated in a two-step process. First, the temperature and heptanol vapor pressure fields inside the condenser were simulated using the finite element modeling software package COMSOL Multiphysics ${ }^{\circledast}$. As only particles detected in the center region of the channel cross section (i.e., viewing window) are used for measurements of particle size and concentration, the temperature $T$ and vapor concentration $C$ were assumed to be independent of $y$ coordinate, and were modeled as 2-dimensional functions of $x$ and $z$ using the following convection-diffusion equations:

$$
\begin{aligned}
& \mathbf{v} \cdot \nabla T=\alpha \nabla^{2} T \\
& \mathbf{v} \cdot \nabla C=D_{v} \nabla^{2} C
\end{aligned},
$$

where $\alpha$ is the thermal diffusivity of air, and $D_{v}$ is the molecular diffusivity of heptanol in air, approximated as $D_{v}=-0.06478+(T /$ $3421.6 \mathrm{~K})+(T / 1664.8 \mathrm{~K})^{2} \mathrm{~cm}^{2} \mathrm{~s}^{-1}$ (Yaws, 2009). The velocity field $\mathbf{v}$ was assumed to be fully developed as above, and has non-zero component only in the $z$ direction described by Eq. (1). For the boundary conditions, the temperature at the entrance of the condenser was set at $24^{\circ} \mathrm{C}$, and $C$ was set as $95 \%$ of the saturation concentration of heptanol (Kemme \& Kreps, 1969):

$$
C_{\text {sat }}(T)=\frac{1}{R T} 953.6 \mathrm{MPa} \cdot \exp \left(\frac{-2893.8 \mathrm{~K}}{T-133.5 \mathrm{~K}}\right)
$$

The temperature of condenser walls was set at $4{ }^{\circ} \mathrm{C}$ (i.e., $20^{\circ} \mathrm{C}$ below the incoming flow temperature), and the heptanol 
concentration at the walls was set at the saturation concentration at the wall temperature, which assumes that the wall surface is coated by condensed heptanol.

The simulated temperature and concentration fields were then imported into MATLAB ${ }^{\mathrm{TM}}$ and interpolated to provide values at any given point in the $x-z$ plane. The growth of the droplets along their trajectories in the condenser was simulated by numerically solving two coupled ordinary differential equations in MATLAB ${ }^{\mathrm{TM}}$. The evolution of droplet diameter $D_{p}$ is governed by (Seinfeld \& Pandis, 2006):

$$
\frac{d D_{p}}{d t}=\frac{4 D_{v}^{\prime} M}{\rho} \frac{\left(C-C_{s}\right)}{D_{p}}
$$

where $M$ and $\rho$ are the molecular weight and density of heptanol, $D_{v}^{\prime}$ is the modified diffusivity of heptanol in air accounting for noncontinuum effects, and is given by $D_{v}^{\prime}=D_{v} \cdot\left[1+\frac{2 D_{v}}{\alpha_{c} D_{p}}\left(\frac{2 \pi M}{R T}\right)^{1 / 2}\right]^{-1}$, where $\alpha_{c}$ is the mass accommodation coefficient, and was assumed as 1 (Seinfeld \& Pandis, 2006). $C_{s}$ represents the equilibrium heptanol concentration at the surface of the growth droplets, and is given by $C_{s}=C_{s a t}\left(T_{s}\right) \exp \left(\frac{4 \gamma M}{\rho R T_{s} D_{p}}\right)$, where $\gamma$ is the surface tension of heptanol, and $T_{s}$ is the temperature at droplet surface, which is governed by (Chang \& Davis, 1974):

$$
\frac{d T_{s}}{d t}=\frac{3}{c_{p} \rho D_{p}}\left(H_{v a p} \rho \frac{d D_{p}}{d t}-4 k_{v}^{\prime} \frac{T_{s}-T}{D_{p}}\right)
$$

where $c_{p}$ and $H_{v a p}$ are the heat capacity and heat of vaporization of heptanol, respectively. $k_{v}^{\prime}$ represents modified thermal conductivity of air and is calculated as $k_{v}^{\prime}=k_{v}\left[1+\frac{2 k_{v}}{\alpha_{T} D_{p} \rho_{a} c_{p, a}}\left(\frac{2 \pi M_{a}}{R T}\right)^{1 / 2}\right]^{-1}$, where $M_{a}, \rho_{a}, c_{p, a}$, and $k_{\nu}$ are the molecular weight, density, heat capacity, and (continuum limit) thermal conductivity of air, respectively. $\alpha_{T}$ is thermal accommodation coefficient, and was assumed to be unity (Seinfeld \& Pandis, 2006). In Eqs. (10) and (11), $C$ and $T$ are the vapor concentration and temperature fields simulated using COMSOL Multiphysics ${ }^{\circledR}$ in the first step. For the simulation of droplet growth, the Brownian motion of particles inside the condenser before activation was neglected, and because there is no electric field in the condenser, the positions of growing droplets as they move along flow streamlines are given by $x=x_{0}, z=u_{z}\left(x_{0}\right) t$, where $x_{0}$ is the initial $x$ position as particles enter the condenser.

The saturation ratio of heptanol and droplet growth along the droplet trajectory (i.e., as a function of $z$ ) are shown in Fig. 8 for different $x$ positions. The entrance of the condenser is represented by $z=0$, and droplets are illuminated and imaged at $z=30.7 \mathrm{~cm}$. Note the saturation ratio is independent of $y$ within the viewing window, and has reflective symmetry across the center plane of $x=0.5 a$. Therefore, the results are shown for particle trajectories with $x$ positions ranging from $0.1 a$ to $0.5 a$. For trajectories near the wall (e.g., $x=0.1 a$ ), the saturation ratio reaches a lower plateau after a shorter distance compared to that along trajectories close to the center plane of the channel (i.e., $x=0.5 a$ ). Near the entrance of the condenser, the saturation ratio close to the wall is higher than that in the center of the channel, due to more rapid temperature decrease of the flow near the wall (Fig. 8a). It is worth noting that particles of smaller sizes near the center plane remain unactivated over a substantially longer distance (Fig. 8b-d), leading to increased Brownian displacement therefore broadening of the transfer function as described earlier. For the latter approximately two third of the condenser, the saturation near the center plane of the condenser (i.e., $x=0.5 a$ ) is substantially higher, due to heptanol vapor depletion near the wall. As a result, for particles of the same diameter, those with trajectories near the center plane grow to larger sizes despite a delayed start. The droplet growth and final droplet diameter (i.e., at $z=30.7 \mathrm{~cm}$ ) only strongly depend on $x$ position near the condenser walls. For particles with same initial diameter, the final droplet diameter exhibits minor variations within the viewing window (i.e., $0.2 a \leq x \leq 0.5 a$ given the symmetry). For example, the final droplet diameters range from 1.9 to $2.3 \mu \mathrm{m}$ for particles with diameter of $50 \mathrm{~nm}$ (Fig. 9). Along the same particle trajectory (i.e. $x$ position), the final droplet diameter is also relatively insensitive to the initial particle diameter ranging from 8 to $600 \mathrm{~nm}$, as expected for condensational droplet growth. The final droplet diameter is greater than $1.8 \mu \mathrm{m}$ within the viewing window for particles as small as $8 \mathrm{~nm}$, suggesting these droplets can be reliably detected optically.

\section{Summary}

A Fast Integrated Mobility Spectrometer employing a spatially varying electric field is developed. In this first of a series of two papers, we present numerical simulations of the characteristics and performances of the FIMS. The spatially varying electric field and particle trajectories inside the FIMS were simulated, with Brownian motion of particles before activation in the condenser taken into account. The simulation results indicate that the FIMS is capable of measuring aerosol size distribution from 8 to $600 \mathrm{~nm}$. Particle Brownian diffusion has minor impact on the transfer function for particles with diameters of $100 \mathrm{~nm}$ or larger, while leads to substantial broadening of transfer function and reduction in mobility resolution for particles with diameters less than $50 \mathrm{~nm}$. For the entire size range of 8 to $600 \mathrm{~nm}$, simulated FIMS resolutions are generally comparable to those of a typical TSI SMPS operated for a similar size range. The growth of particles inside the condenser was simulated, the results show the final droplet diameter are fairly insensitive to the particle position detected within the viewing window or the initial particles diameters ranging from 8 to $600 \mathrm{~nm}$. The final droplet diameter at the point of detection is greater than $1.8 \mu \mathrm{m}$ within the viewing window for particles as small as $8 \mathrm{~nm}$, suggesting the grown droplets can be reliably detected optically. 


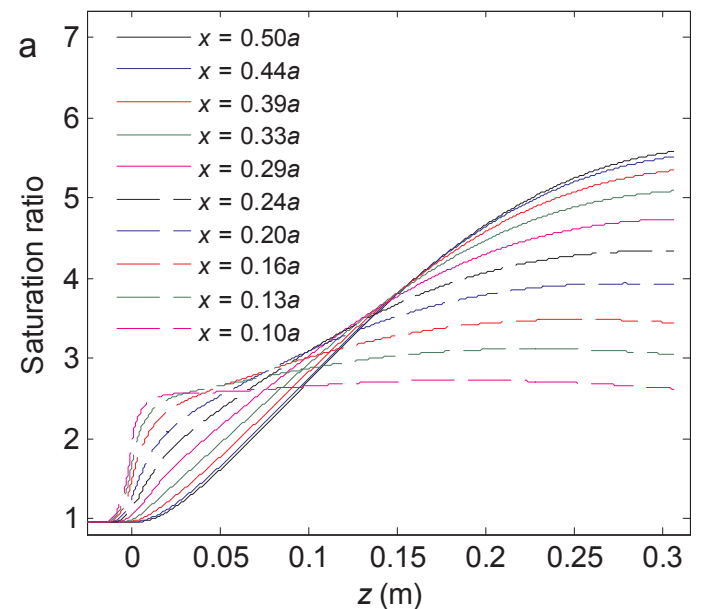

Initial particle diameter: $10 \mathrm{~nm}$

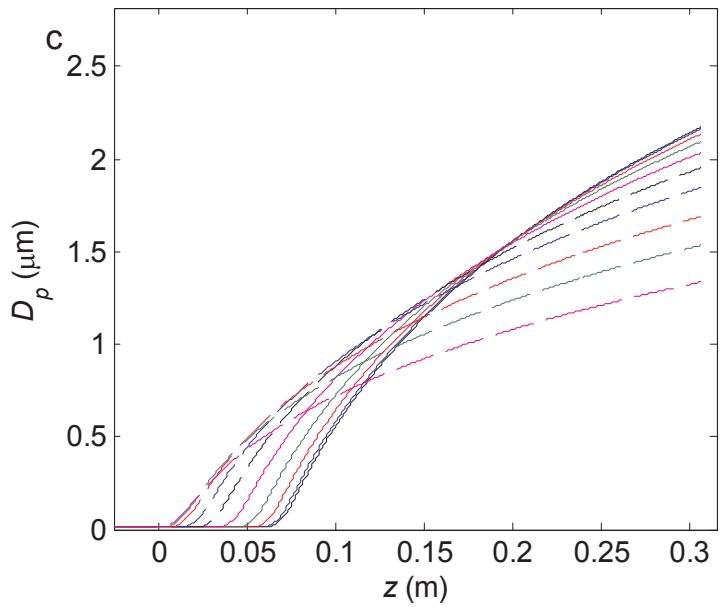

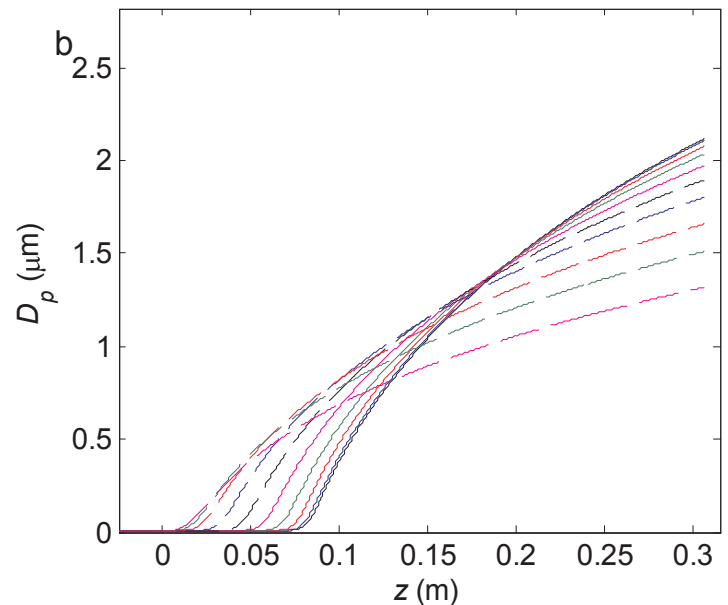

Initial particle diameter: $100 \mathrm{~nm}$

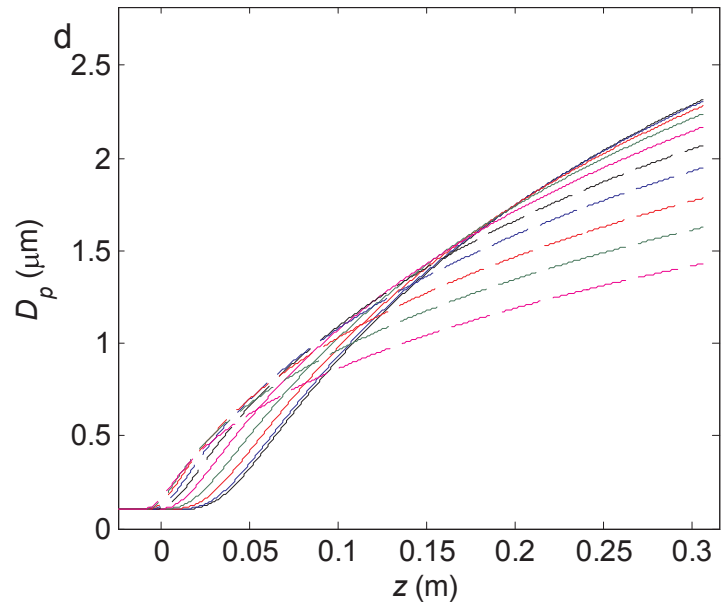

Fig. 8. (a) Saturation ratio along particle trajectories inside the condenser for different $x$ positions, and diameters of growing droplets along trajectories starting with different $x$ positions for particles with initial diameters of (b) 8 , (c) 10 , (d) $100 \mathrm{~nm}$, respectively. The entrance of the condenser is represented by $z=0$, and droplets are illuminated and imaged at $z=30.7 \mathrm{~cm}$.

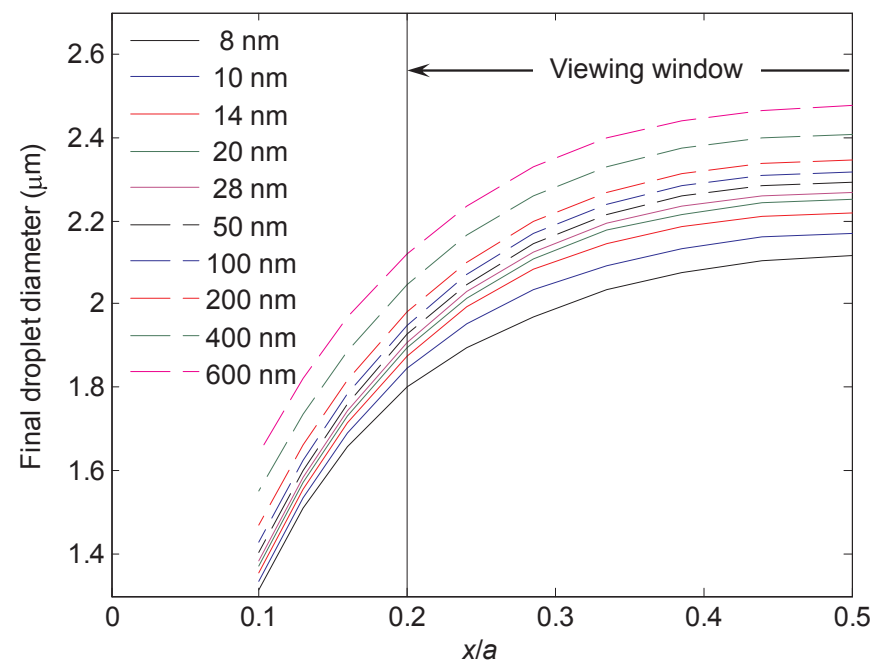

Fig. 9. The final droplet diameter at the point of detection as a function of trajectory position $x / a$ for particles with diameters ranging from 8 to 600 nm. 


\section{Acknowledgments}

This work was supported by the U.S. Department of Energy's Small Business Innovation Program under contract DE-SC0006312, and Atmospheric System Research Program (Office of Science, OBER) under contract DE-AC02-98CH10886.

\section{References}

Barmpounis, K., Maisser, A., Schmidt-Ott, A., \& Biskos, G. (2016). Lightweight differential mobility analyzers: Toward new and inexpensive manufacturing methods. Aerosol Science and Technology, 50(1), http://dx.doi.org/10.1080/02786826.2015.1130216.

Biskos, G., Reavell, K., \& Collings, N. (2005). Description and theoretical analysis of a differential mobility spectrometer. [Article]. Aerosol Science and Technology, 39(6), 527-541. http://dx.doi.org/10.1080/027868291004832.

Chang, R., \& Davis, E. J. (1974). Interfacial conditions and evaporation rates of a liquid droplet. Journal of Colloid and Interface Science, 47(1), 65-76. http://dx.doi. org/10.1016/0021-9797(74)90080-0.

Chen, D. R., Pui, D. Y. H., Hummes, D., Fissan, H., Quant, F. R., \& Sem, G. J. (1998). Design and evaluation of a nanometer aerosol differential mobility analyzer (NanoDMA). Journal of Aerosol Science, 29(5-6), 497-509. http://dx.doi.org/10.1016/s0021-8502(97)10018-0.

Ermak, D. L. (1975). Computer simulation of charged particles in solution. I. Technique and equilibrium properties. Journal of Chemical Physics, 62, 4189-4196.

Flagan, R. C. (2004). Opposed Migration Aerosol Classifier (OMAC). Aerosol Science and Technology, 38(9), 890-899.

Han, H. S., Chen, D. R., Pui, D. Y. H., \& Anderson, B. E. (2000). A nanometer aerosol size analyzer (nASA) for rapid measurement of high-concentration size distributions. [Article]. Journal of Nanoparticle Research, 2(1), 43-52. http://dx.doi.org/10.1023/a:1010014109495.

Jiang, J. K., Attoui, M., Heim, M., Brunelli, N. A., McMurry, P. H., Kasper, G., \& Mouret, G. (2011). Transfer Functions and Penetrations of Five Differential Mobility Analyzers for Sub-2 nm Particle Classification. Aerosol Science and Technology, 45(4), 480-492. http://dx.doi.org/10.1080/02786826.2010.546819.

Johnson, T., Caldow, R., Pocher, A., Mirme, A., \& Kittelson, D. B. (2004). A new electrical mobility particle sizer spectrometer for engine exhaust particle measurements. SAE Technical Paper, 2004-2001-1341. http://dx.doi.org/10.4271/2004-01-1341.

Kemme, H. R., \& Kreps, S. I. (1969). Vapor pressure of primary n-Alkyl chlorides and alcohols. Journal of Chemical and Engineering Data, 14(1), http://dx.doi.org/10. 1021/je60040a011.

Kulkarni, P., \& Wang, J. (2006). New fast integrated mobility spectrometer for real-time measurement of aerosol size distribution - I: Concept and theory. Journal Aerosol Science, 37(10), 1303-1325.

Kulkarni, P., \& Wang, J. (2006). New fast integrated mobility spectrometer for real-time measurement of aerosol size distribution: II. Design, calibration, and performance characterization. Journal of Aerosol Science, 37(10), 1326-1339.

Oberreit, D. R., McMurry, P. H., \& Hogan, C. J. (2014). Mobility Analysis of $2 \mathrm{~nm}$ to $11 \mathrm{~nm}$ Aerosol Particles with an Aspirating Drift Tube Ion Mobility Spectrometer. Aerosol Science and Technology, 48(1), 108-118. http://dx.doi.org/10.1080/02786826.2013.861893.

Olfert, J. S., \& Wang, J. (2009). Dynamic Characteristics of a Fast-Response Aerosol Size Spectrometer. Aerosol Science and Technology, $43(2), 97-111$.

Olfert, J. S., Kulkarni, P., \& Wang, J. (2008). Measuring aerosol size distributions with the fast integrated mobility spectrometer. Journal of Aerosol Science, 39(11), 940-956.

Rosser, S., \& de la Mora, J. F. (2005). Vienna-type DMA of high resolution and high flow rate. Aerosol Science and Technology, 39(12), 1191-1200. http://dx.doi.org/10. $1080 / 02786820500444820$.

Seinfeld, J. H., \& Pandis, S. N. (2006). Atmospheric chemistry and physics (2nd ed). Hoboken: John Wiley \& Sons, Inc.

Song, D. K., \& Dhaniyala, S. (2007). Nanoparticle cross-flow differential mobility analyzer (NCDMA): theory and design. Journal of Aerosol Science, 38(9), 964-979. http://dx.doi.org/10.1016/j.jaerosci.2007.07.004.

Steer, B., Gorbunov, B., Muir, R., Ghimire, A., \& Rowles, J. (2014). Portable planar DMA: development and tests. Aerosol Science and Technology, 48(3), 251-260. http://dx.doi.org/10.1080/02786826.2013.868863.

Tammet, H., Mirme, A., \& Tamm, E. (2002). Electrical aerosol spectrometer of Tartu University. Atmospheric Research, 62(3-4), 315-324. http://dx.doi.org/10.1016/ s0169-8095(02)00017-0.

Wang, J. (2009). A fast integrated mobility spectrometer with wide dynamic size range: Theoretical analysis and numerical simulation. Journal Aerosol Science, 40(10), 890-906.

Wang, J., McNeill, V. F., Collins, D. R., \& Flagan, R. C. (2002). Fast mixing condensation nucleus counter: Application to rapid scanning differential mobility analyzer measurements. [Article]. Aerosol Science and Technology, 36(6), 678-689. http://dx.doi.org/10.1080/02786820290038366.

Wang, J., Storey, J., Domingo, N., Huff, S., Thomas, J., \& West, B. (2006). Studies of diesel engine particle emissions during transient operations using an engine exhaust particle sizer. [Article]. Aerosol Science and Technology, 40(11), 1002-1015. http://dx.doi.org/10.1080/02786820600919408.

Wang, S. C., \& Flagan, R. C. (1990). Scanning electrical mobility spectrometer. Aerosol Science and Technolnology, 13(2), 230-240.

Yaws, C. L. (2009). Transport properties of chemicals and hydrocarbons (1st ed). Elsevier Inc. 